Service social

\title{
Modes de résolution de problèmes des jeunes familles immigrantes : résultats de recherche
}

\section{Sylvie Fortin et Gisèle Legault}

Volume 43, numéro 2, 1994

Enfants, parents, intervenants

URI : https://id.erudit.org/iderudit/706659ar

DOI : https://doi.org/10.7202/706659ar

Aller au sommaire du numéro

\section{Éditeur(s)}

École de service social de l'Université Laval

ISSN

1708-1734 (numérique)

Découvrir la revue

Citer cet article

Fortin, S. \& Legault, G. (1994). Modes de résolution de problèmes des jeunes familles immigrantes : résultats de recherche. Service social, 43(2), 97-127. https://doi.org/10.7202/706659ar
Résumé de l'article

Cet article présente les résultats d'une recherche de type exploratoire-descriptive, conduite auprès de 297 jeunes familles immigrantes et de 40 intervenants, sur les problèmes sociaux et culturels que les familles rencontrent et sur leurs modes de résolution de ces problèmes. Dans le secteur social, les difficultés prioritaires des familles sont principalement liées à la problématique du travail. Dans le secteur culturel, l'isolement et l'ennui sont au premier rang des problèmes prioritaires, suivis des problèmes de garde des enfants et des modalités d'éducation en pays d'accueil. Les intervenants jugent plutôt prioritaires des problèmes de dynamique de couple. Alors qu'à la fois familles et intervenants expriment des souhaits de consultation du réseau formel de services pour les problèmes sociaux, les familles préfèrent le réseau informel pour les problèmes culturels. Les difficultés d'accessibilité et de compatibilité des services sont perçues comme plus considérables par les intervenants que par les familles. 


\section{Modes de résolution de problèmes des jeunes familles immigrantes Résultats de recherche}

SYLVIE FORTIN

Agente de recherche Direction de la santé publique, Montréal-Centre

GISÈLE LEGAULT

Professeure titulaire École de service social, Université de Montréal

\section{INTRODUCTION}

Depuis des décennies, le Québec accueille annuellement plus de 30000 immigrants. Alors que ces immigrants venaient d'Europe au cours des années 60 et 70, ils sont maintenant en majorité originaires de pays en voie de développement (Groupe de recherche Ethnicité et Société, GRES,1992). Cette migration est aussi essentiellement urbaine et montréalaise, puisque $90 \%$ des immigrants élisent domicile à Montréal où la population immigrante atteint $16 \%$ (Baillargeon, 1989).

L'adaptation de ces immigrants est une question complexe qui préoccupe plusieurs niveaux de la société québécoise, du ministère des Communautés culturelles et de l'Immigration (MCCl) aux 
organismes de santé et de services sociaux du réseau des affaires sociales. Les Centres locaux de services communautaires (CLSC), responsables des services de première ligne de ce réseau, sont conscients des nombreux besoins en santé et en services sociaux de ces familles, tout particulièrement au cours de leurs premières années d'adaptation. Ils constatent aussi les difficultés qu'il y a à rejoindre plusieurs de ces familles et à leur offrir des services appropriés, accessibles et culturellement compatibles.

Consciente de ces réalités, notre équipe ${ }^{1}$ a effectué une recherche (Heneman et al., 1994) sur les besoins prioritaires en santé et services sociaux des jeunes familles immigrantes et sur les réponses du réseau à ces besoins.

Notre recherche a été une entreprise menée de concert par la Régie régionale de la santé et des services sociaux de Montréal, la Direction de la santé publique et l'École de service social de I'Université de Montréal à laquelle trois CLSC de la région ouest de Montréal se sont associés dès le début, soit les CLSC Côte-des-Neiges, SaintLaurent et Bordeaux-Cartierville. Ces établissements desservent des populations dont les concentrations multiethniques étaient respectivement, en 1986, de $35 \%, 33 \%$ et $41 \%$ (Choinière, 1990). Malgré ces concentrations multiethniques, les CLSC constatent que les différents services, tels que les services courants, la périnatalité et les services sociaux, rejoignent dans une proportion inégale les familles récemment immigrées (Gravel et Guay, 1990; Desjardins, 1990).

La recherche, subventionnée par Santé et Bien-être Canada, visait les objectifs suivants: préciser le profil sociodémographique des familles récemment immigrées (moins de cinq ans), préciser leurs difficultés $\mathrm{d}^{\prime}$ adaptation en définissant les problèmes de santé, problèmes sociaux et culturels prioritaires qu'ils rencontrent, préciser leurs modes de résolution de ces problèmes par l'utilisation des réseaux formel et informel de services et, finalement, relever les difficultés de disponibilité, accessibilité et compatibilité culturelle des services offerts.

Nous avons exposé ailleurs (Legault et Fortin, 1994) notre cadre conceptuel et certains aspects de notre devis de recherche ${ }^{2}$. Nous nous concentrerons donc, dans le présent article, sur la méthode utilisée, sur les principaux résultats des secteurs social et culturel et sur les implications d'une telle étude en matière d'intégration des immigrants et du rôle des agents d'aide à cette intégration.

1. Notre équipe de recherche est constituée aussi de B. Heneman, S. Gravel et E. Alvarado avec la collaboration de D. Groleau.

2. L'article cité traite, en plus du devis explicité ici, des variables à l'étude pour les familles et les intervenants. On s'y référera pour plus de détails. 


\section{DEVIS DE RECHERCHE ET MÉTHODOLOGIE}

L'étude, de type exploratoire-descriptive, a été élaborée à partir du processus de résolution de problèmes tel que vécu par un échantillon de familles d'immigration récente de la région de Montréal ayant de jeunes enfants et tel que perçu par un échantillon $d^{\prime}$ intervenants auprès de cette population.

\section{Population à l'étude et échantillonnage}

Familles. La population à l'étude a été constituée de familles récemment immigrées (moins de cinq ans), ayant au moins un enfant d'âge préscolaire (moins de cinq ans) et résidant sur les territoires des trois CLSC mentionnés. Afin d'établir cette population, nous avons demandé à la Régie de l'assurance-maladie du Québec (RAMQ) de nous constituer un fichier de toutes les nouvelles inscriptions, entre juillet 1989 et juin 1992, de femmes adultes nées à l'extérieur du Canada et correspondant aux critères énoncés précédemment. Ce fichier comptait 1890 familles. Pour obtenir des intervalles de confiance avec une étendue de $10 \%$ au niveau de confiance $(1-\mathrm{a})=95 \%$ pour des proportions pertinentes, un échantillon d'approximativement 320 familles était nécessaire. Comme on prévoyait que le pourcentage d'acceptation à I'entrevue serait très élevé en raison des objectifs de la recherche, un premier échantillon d'environ 380 familles a été tiré.

L'échantillonnage des familles s'est effectué de façon systématique à partir de la liste fournie par la RAMQ. Un total de 376 familles ont été choisies dans un premier temps. En raison du nombre imprévu de familles introuvables, soit 101, une deuxième série de familles a dû être choisie; finalement, 297 entrevues ont été complétées. Le taux de refus de la population échantillonnée et rejointe a été de $15 \%$.

\section{TAbleAu 1}

\section{Échantillon des familles}

\begin{tabular}{lcccccc}
\hline & $\begin{array}{c}\text { Taille de } \\
\text { l'échantillon }\end{array}$ & Introuvable & Refus & $\begin{array}{c}\text { Acceptation } \\
\text { par la poste }\end{array}$ & $\begin{array}{c}\text { Acceptation } \\
\text { par téléphone }\end{array}$ & $\begin{array}{c}\text { Entrevues } \\
\text { complétées }\end{array}$ \\
\hline Liste 1 (08-92) & 376 & 101 & 40 & 88 & 147 & 235 \\
Liste 2 (11-92) & 126 & 50 & 14 & 10 & 52 & 62 \\
Total & $\mathbf{5 0 2}$ & $\mathbf{1 5 1}$ & $\mathbf{5 4}$ & $\mathbf{9 8}$ & $\mathbf{1 9 9}$ & $\mathbf{2 9 7}$ \\
\hline
\end{tabular}


Intervenants. L'autre population à l'étude a été celle des intervenants travaillant dans les trois CLSC associés à la recherche et dans les organismes communautaires voués à l'installation des familles immigrantes sur ces mêmes territoires. Afin d'établir cette deuxième population, une liste de tous les intervenants du secteur médical et social travaillant auprès des familles récemment immigrées a été constituée par les trois CLSC. Cette liste comprenait un total de 70 intervenants, soit 55 du réseau public et 15 du réseau communautaire. Une deuxième source d'intervenants du réseau public, secteur social, provenait du Service migrants-immigrants (maintenant affilié au CLSC Parc Extension) desservant la clientèle du Montréal métropolitain. Ce service totalisait 17 intervenants travaillant exclusivement auprès des familles immigrantes. Ainsi, au mois de mars 1993, notre population totale $d^{\prime}$ intervenants comprenait 94 personnes.

Tableau 2

Échantillon des intervenants

\begin{tabular}{|c|c|c|c|c|}
\hline & & & $\begin{array}{c}\text { Taille de la } \\
\text { population } \\
\text { dans la strate }\end{array}$ & $\begin{array}{c}\text { Entrevues } \\
\text { complétées }\end{array}$ \\
\hline \multirow[t]{3}{*}{ CLSC } & Côte-des-Neiges & $\begin{array}{l}\text { médical } \\
\text { social }\end{array}$ & $\begin{array}{l}22 \\
10\end{array}$ & $\begin{array}{l}9 \\
6\end{array}$ \\
\hline & Saint-Laurent & $\begin{array}{l}\text { médical } \\
\text { social }\end{array}$ & $\begin{array}{l}9 \\
3\end{array}$ & $\begin{array}{l}2 \\
3\end{array}$ \\
\hline & Bordeaux-Cartierville & $\begin{array}{l}\text { médical } \\
\text { social }\end{array}$ & $\begin{array}{l}7 \\
4\end{array}$ & $\begin{array}{l}4 \\
2\end{array}$ \\
\hline \multirow[t]{4}{*}{$\begin{array}{l}\text { Organismes } \\
\text { communautaires }\end{array}$} & $\begin{array}{l}\text { Territoire du } \\
\text { CLSC Côte-des-Neiges }\end{array}$ & & 5 & 3 \\
\hline & $\begin{array}{l}\text { Territoire du } \\
\text { CLSC Saint-Laurent }\end{array}$ & & 5 & 3 \\
\hline & $\begin{array}{l}\text { Territoire du CLSC } \\
\text { Bordeaux-Cartierville }\end{array}$ & & 5 & 4 \\
\hline & Multiethnique & & 7 & 0 \\
\hline CSSMM & & social & 17 & 4 \\
\hline Total & & & 94 & 40 \\
\hline
\end{tabular}

Avec l'objectif d'obtenir un nombre égal d'intervenants des secteurs médical et social, 40 intervenants furent interviewés. Nous avons choisi aléatoirement des intervenants du réseau CLSC par strate où chaque strate a été définie par une combinaison CLSC / secteur (médical ou social) pour un total de six strates (trois CLSC $x$ deux secteurs par 
CLSC). Les intervenants du réseau communautaire furent aussi échantillonnés de façon aléatoire par strate définie par les CLSC, car la catégorisation par secteur n'était pas possible dans le réseau communautaire. Les intervenants du Service migrants-immigrants échantillonnés furent désignés par le responsable en fonction de nul autre critère que leur disponibilité, le choix étant arbitrairement considéré comme aléatoire.

\section{Procédure de collecte de données}

Le répondant choisi a été la mère, parce que nous la croyions la plus à même de traiter des sujets à l'étude. Les entrevues ont été effectuées à domicile pour les familles et au lieu de travail pour les intervenants. Elles ont eu respectivement une durée d'une heure et demie et d'une heure. Afin de couvrir l'ensemble des langues parlées par les familles composant notre échantillon, nous avons eu recours à dix-huit intervieweuses et à trois interprètes. Plusieurs intervieweuses étant bilingues ou trilingues, le recours à des interprètes a été considérablement réduit.

L'instrument de recherche a été le questionnaire composé essentiellement de questions fermées avec quelques questions ouvertes dans le but de préciser certains éléments de contenu. Les questions étaient divisées en quatre sections portant sur le profil socio-démographique des familles et des intervenants, puis sur les secteurs sanitaire, social et culturel.

Le questionnaire a été élaboré à partir de la recension des écrits, des avis des professionnels du Département de santé communautaire de I'hôpital Sainte-Justine et d'un guide d'entrevue utilisé dans une recherche antérieure auprès d'intervenants sociaux et communautaires (Legault et Lafrenière, 1992).

\section{Analyse et traitement des données}

L'analyse des données a été faite à partir du construit théorique (tableau 3) où l'on retrouve la séquence de problèmes décrits et hiérarchisés et le cheminement de consultation à partir des personnes souhaitées, de leur disponibilité, de l'accessibilité des personnes effectivement consultées et de la compatibilité culturelle des services offerts. Mentionnons que l'analyse des données des familles et des intervenants s'est faite en parallèle, afin d'effectuer une analyse comparative des perceptions des deux groupes. Les perspectives des unes et des autres diffèrent toutefois. Alors que les témoignages des familles sont liés à 
leur situation réelle au cours de l'année précédant l'entrevue (19921993), ceux des intervenants se basent sur une situation hypothétique se rapportant à l'ensemble de leur clientèle, par le truchement de leur appartenance institutionnelle.

Nous nous sommes finalement servis du logiciel SPSS pour traiter nos données et ainsi obtenir des distributions de fréquences et des tableaux croisés (tableau 3).

\section{PROFIL SOCIODÉMOGRAPHIQUE DES FAMILLES ET DES INTERVENANTS}

Les familles rencontrées avaient de jeunes enfants ( $57 \%$ des enfants répertoriés ont moins de cinq ans) et sont pour la plupart arrivées au Québec au cours des quatre dernières années (87\% des répondantes et $66 \%$ des conjoints). Leur départ du pays d'origine a été dans $75 \%$ des cas planifié et, parmi les raisons ayant motivé ce départ (les répondantes pouvaient choisir plusieurs raisons), la raison prioritaire, pour $53 \%$ des familles, a été d'améliorer la vie future des enfants. Des raisons politiques sont invoquées dans $39 \%$ des cas et des raisons économiques dans $29 \%$ des cas. Ces familles sont d'origine urbaine dans $84 \%$ des cas, elles ont le statut de résidents permanents et ont en moyenne 2,14 enfants. Les pays d'origine sont principalement le Liban pour $37 \%$ des répondantes, le Salvador et le Maroc pour $5 \%$, la Syrie pour $4 \%$ et l'Égypte pour $3 \%$. En matière de connaissance des langues officielles du pays d'accueil, $47 \%$ des répondantes et $52 \%$ des conjoints parlent couramment le français, alors que $35 \%$ et $56 \%$ respectivement parlent I'anglais. Les langues les plus couramment parlées à la maison sont l'arabe dans $48 \%$ des cas, le français dans $21 \%$, I'espagnol dans $10 \%$ et l'anglais dans $8 \%$ des cas. Les répondantes sont en majorité de religion chrétienne $(63 \%)$ et ont un niveau de scolarité élevé : $44 \%$ ont plus de 13 ans d'études et $69 \%$, plus de 11 ans. Il en est de même des conjoints (53\% et $72 \%$ ). Alors que $24 \%$ des répondantes sont sur le marché du travail, $61 \%$ sont des travailleuses au foyer; les conjoints sont, quant à eux, au travail dans $51 \%$ des cas et chômeurs dans $34 \%$ des cas. Ces familles ont finalement un niveau socio-économique qui se situe sous le seuil de la pauvreté dans $78 \%$ des cas et sous le seuil de grande pauvreté dans $59 \%$ des $\operatorname{cas}^{3}$.

3. Les seuils de pauvreté sont ceux du Conseil national de bien-être social en 1992 et ceux de grande pauvreté correspondent à $60 \%$ du seuil de pauvreté, ainsi qu'ils ont été qu'établis par Wilkins (1985). 


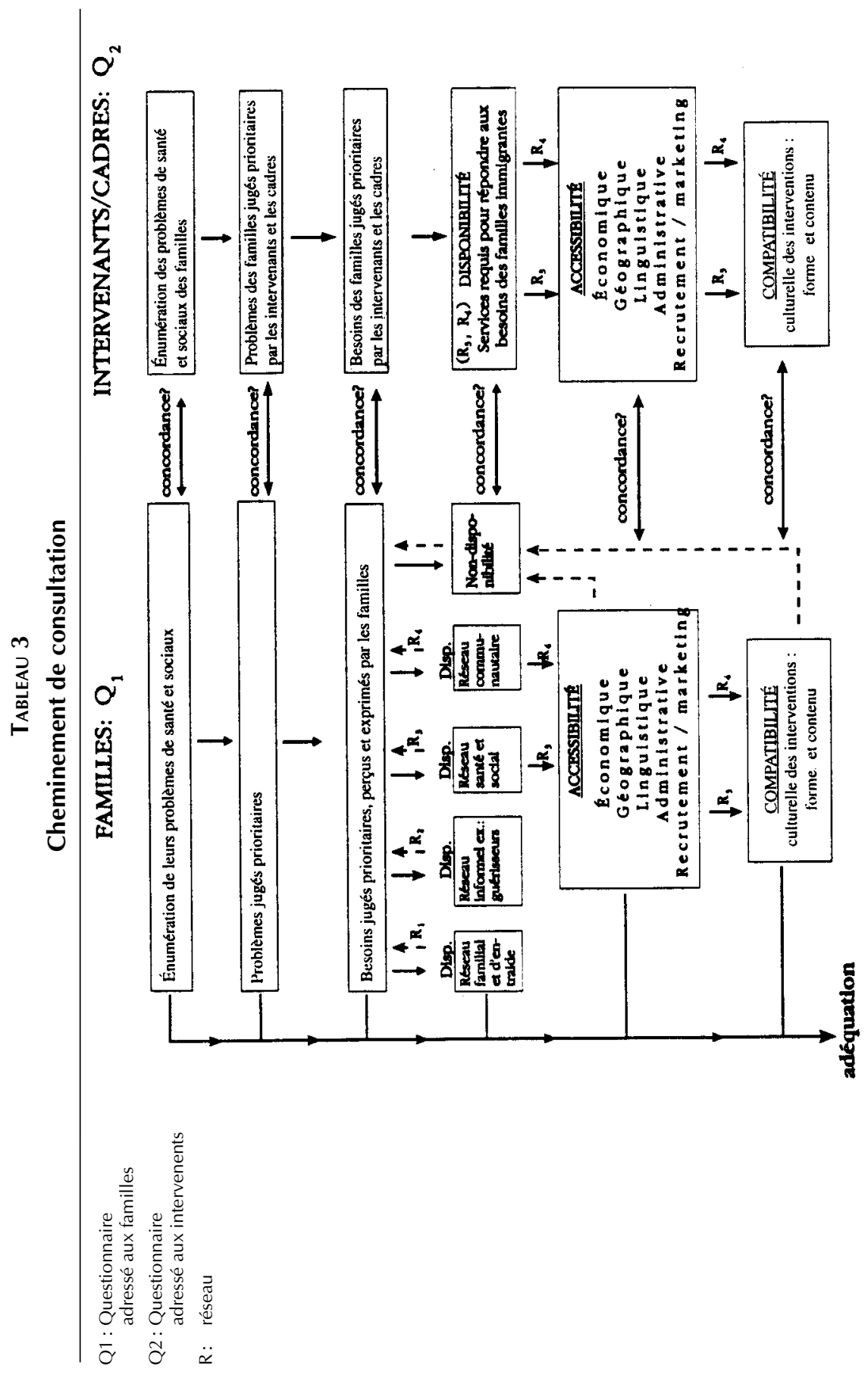


Les intervenants rencontrés sont en majorité des femmes (33 sur 40). Elles se répartissent assez également entre le secteur de la santé et le secteur social et leur formation a été obtenue principalement en études infirmières et en travail social. Ce sont des professionnelles d'expérience (près de la moitié ont plus de cinq ans de pratique), rodées à la pratique auprès de clientèles immigrantes $(65 \%$ consacrent les trois quarts de leur temps à une clientèle immigrante). Elles ont un profil ethnoculturel varié et parlent plusieurs langues $(40 \%$, deux langues et $20 \%$, trois langues et plus). Soixante-quinze pour cent d'entre elles ont reçu et $45 \%$ continuent de recevoir une formation à la pratique interculturelle au moment de l'enquête.

\section{HIÉRARCHISATION DES PROBLÈMES}

Les choix méthodologiques de l'étude nous permettent d'obtenir des données représentatives quant aux problèmes des familles et quant à leurs modes de résolution. Il est ainsi possible d'étendre les résultats obtenus à la population des jeunes familles immigrantes des territoires visés et, plus prudemment, à la population des jeunes familles immigrantes de la région de Montréal. Il faut cependant garder en mémoire les particularités locales des CLSC concernés, dont la sur-représentation de la population d'origine libanaise sur les territoires de ces CLSC.

\section{Problèmes sociaux prioritaires ${ }^{4}$}

Comme nous I'indique le tableau 4, tant chez les familles que chez les intervenants, ce sont les problèmes liés au travail (en trouver, remplir les conditions pour y accéder) qui dominent en matière de problèmes sociaux prioritaires. Les intervenants placent toutefois les problèmes financiers au premier rang des problèmes prioritaires. Il va de soi que ces deux types de problèmes sont fortement liés.

4. Une liste de problèmes sociaux était jointe au questionnaire. À l'aide de cette liste, la répondante indiquait quels problèmes elle avait rencontrés au cours de la dernière année, pour ensuite préciser les deux qu'elle estimait prioritaires. Cette liste comprenait: des problèmes financiers; des problèmes de communication au travail, dans les services, dans les commerces et les magasins; des problèmes à trouver du travail ou des problèmes liés aux conditions de travail, à la reconnaissance de diplômes étrangers, à la reconnaissance d'expérience antérieure de travail, à la déqualification professionnelle; des problèmes liés au statut d'immigration ; des problèmes à trouver un logement, liés au coût, à la dimension ou aux conditions de ce logement; des problèmes de discrimination / racisme autour de la recherche d'un travail, d'un logement, dans les services publics, dans les commerces et magasins, dans l'utilisation de l'anglais au Québec. 
Tableau 4

Hiérarchisation des problèmes sociaux

par les familles et les intervenants

\begin{tabular}{lcc}
\hline Problèmes sociaux jugés prioritaires & $\begin{array}{c}\text { Rang selon } \\
\text { familles }\end{array}$ & $\begin{array}{c}\text { Rang selon } \\
\text { intervenants }\end{array}$ \\
\hline Problèmes liés au manque de travail & 1 & 2 \\
Problèmes de communication & 2 & 4 \\
Problèmes liés à l'accès au travail & 3 & $*$ \\
Problèmes financiers & 4 & 1 \\
Problèmes de logement & 5 & 5 \\
Problèmes de discrimination & 6 & $*$ \\
Problèmes liés au statut d'immigration & 7 & 3 \\
\hline
\end{tabular}

* Ces problèmes n’ont pas été jugés prioritaires.

L'intégration socio-économique des immigrants est un volet crucial de leur intégration globale, comme le soulignait le ministère des Communautés culturelles et de l'Immigration (MCCI, 1990) dans un document sur le sujet. Ce document vient expliciter les dimensions de l'intégration des immigrants par rapport à la politique officielle du Ministère. L'intégration, d'abord présentée comme un processus à long terme et multidimensionnel, est ensuite précisée selon l'intégration socio-économique, linguistique, culturelle, institutionnelle, personnelle, communautaire et sociale. L'intégration socio-économique est présentée comme plus ou moins complexe selon les acquis prémigratoires et les obstacles structurels de la société d'accueil, tels une récession économique et un haut taux de chômage. Plusieurs des familles de notre échantillon se situent dans une phase d'adaptation fonctionnelle, associée aux premières années d'établissement dans un nouveau pays. Elles y rencontrent donc des obstacles structurels tels que ceux mentionnés, tout en ayant, pour beaucoup, des acquis prémigratoires intéressants. C'est ce qui, croyons-nous, leur fait reconnaître et juger prioritaire ce type de problème social.

Les familles de notre échantillon estiment également prioritaires certains problèmes que nous avons regroupés sous le vocable de problèmes d'accès au travail. Nous avons inclus dans cette catégorie les problèmes de non-reconnaissance de l'expérience de travail à l'extérieur du Canada, de déqualification professionnelle et de conditions de travail, faisant en cela référence à ce que Bibeau et al. (1992) désignent sous le concept d'organisation hiérarchisée du travail. 
Si nous comparons nos données avec celles de l'étude de Renaud et al. (1993) effectuée à Montréal sur le processus d'établissement d'immigrants récents, il semble que la situation d'emploi qui y est décrite soit quelque peu meilleure. Ainsi on note que «l'obtention d'un premier emploi se fait essentiellement dès la première année d'établissement » (Renaud et al., 1993: 11) et que "les femmes prennent presque une année avant que leur situation d'emploi se stabilise autour de $50 \%$, alors que la situation des hommes se stabilise après la semaine 10, à une probabilité d'environ 65\%» (Renaud et al., 1993: 21). Cette situation peut se comparer à notre échantillon où $51 \%$ des conjoints travaillent alors que $34 \%$ sont en chômage et que la durée médiane de présence au Canada de ceux-ci est de deux ans et demi. Peut-être le fait que les familles reconnaissent et jugent prioritaires les problèmes de travail dans le secteur social est-il dû autant à un travail obtenu qui est en-deçà des compétences des personnes qu'à l'absence de travail comme telle.

Il faut aussi noter que $62 \%$ des répondantes sont travailleuses au foyer au Québec comparativement à $17 \%$ dans le pays d'origine. Elles sont maintenant sur le marché du travail dans une proportion de $24 \%$ contre $67 \%$ dans le pays d'origine. Il y a donc, au Québec, une baisse de la présence des femmes sur le marché du travail qui peut, en partie, s'expliquer par la présence d'enfants d'âge préscolaire. Cette réalité, jumelée avec une possible insatisfaction liée au type d'emploi occupé ou au doute quant à la possibilité de trouver du travail, leur fait d'autant plus juger prioritaire ce type de problème social.

Une deuxième constatation au chapitre des problèmes sociaux a trait à l'importance accordée par les familles de notre échantillon (deuxième rang) autant que par les intervenants (quatrième rang) aux problèmes de communication. Comme le soulignait Beiser et al. (1988: 29), «il est impossible de vraiment s'adapter à une culture si on n'en connaît pas la langue». Ce rapport attirait l'attention sur ce type de problème, estimant que de nombreux problèmes sociaux et de santé y sont directement attribuables, les individus qui ne maîtrisent pas l'une des deux langues ayant le sentiment d'être des marginaux plutôt que des membres à part entière de la société. Notre échantillon comporte toutefois un pourcentage élevé de personnes $(65 \%$ des répondantes et $76 \%$ des conjoints) qui maîtrisent soit l'anglais, soit le français. II est par conséquent paradoxal que les problèmes de communication soient placés au deuxième rang des priorités. Cela l'est moins pour les intervenants qui rencontrent une large clientèle d'immigrants et qui sont de ce fait à même d'obser- ver ces difficultés sur une base continue. Peut-être trouve-t-on un facteur explicatif du côté des familles 
lorsqu'on observe que la langue le plus souvent parlée à la maison est I'arabe (dans $48 \%$ des cas). Renaud et al. (1993) observent, tout comme nous, qu'après trois ans la langue le plus fréquemment utilisée à la maison est, dans $67 \%$ des cas, la langue maternelle, autre que française ou anglaise. Le français n'est la langue le plus souvent parlée que par $21 \%$ des familles de I'échantillon. Un autre facteur non négligeable est la politique linguistique du Québec, axée sur le français comme principale langue de communication. Pour les familles qui parlent peu ou pas le français, le fait de vivre à Montréal peut être source de difficultés. Ainsi, bien qu'un pourcentage élevé d'entre elles parlent l'une des deux langues de la société d'accueil, les familles demeurent sans doute peu habiles et mal à l'aise de le faire.

Une troisième constatation a trait aux problèmes de logement que les familles et les intervenants placent au cinquième rang des problèmes sociaux. Ce type de problèmes est fréquent chez les jeunes familles immigrantes. Bibeau et al. (1992:201) mentionnent que les immigrants des années 80 , quoique possédant souvent un très bon niveau de scolarité, constituent une population au statut économique précaire. Des «conditions de vie précaires et le coût élevé des loyers compromettent le droit au logement ou le maintien dans les lieux». Nos résultats abondent dans le même sens, puisque nous avons rencontré précisément une population ayant un bon niveau de scolarité, tout en ayant un statut économique précaire, et où les familles consacrent en moyenne 529,50 \$ par mois à leur logement. Cela se compare assez bien avec les données de l'étude de Renaud et al. (1993: 62) où l'on note que «la moyenne du montant mensuel consacré au logement croît légèrement tout au long de l'enquête. À la première semaine, la moyenne est de 450 \$. Cette valeur est de 548 \$ lors de la semaine 140 , c.-à-d. après trois ans. " Les familles de notre échantillon qui placent les problèmes de logement au cinquième rang estiment sans doute qu'elles consacrent à cet élément une trop grande proportion de leurs maigres revenus ou qu'elles sont confinées à des logements plus petits que ce qu'elles auraient souhaité. Les intervenants pour leur part font référence au problème partagé par beaucoup d'immigrants de trouver un logement adéquat, de la dimension voulue et à un prix adapté à leur situation financière.

Nous voudrions finalement commenter le fait que les intervenants placent au troisième rang les problèmes liés au statut d'immigration, alors que les familles ne situent ces problèmes qu'au septième rang. Les intervenants font sans doute référence aux statuts d'immigration fréquents des années 80 et 90, soit les statuts de la catégorie famille et ceux de requérants au statut de réfugié. Ces catégories, typiques de 
I'immigration des récentes années et probablement sur-représentées dans les clientèles (Labelle, 1989), donnent lieu à différents problèmes dont les intervenants sont conscients, tels que la situation de dépendance dans laquelle sont placés les ressortissants de la catégorie 'familles' et les démarches multiples auxquelles doivent se soumettre les requérants au statut de réfugié, sans oublier le peu de mesures sociales auxquelles ces catégories ont accès pendant ce temps.

Ces problèmes liés au statut d'immigration viennent plus loin dans I'ordre de priorité des familles en raison, croyons-nous, de leur statut particulièrement stable d'immigrants reçus ou de citoyens. En effet, on note que $95 \%$ des répondantes et $97 \%$ des conjoints ont ces statuts. À l'exception du droit de vote pour les immigrants reçus, ces statuts donnent accès à la totalité des mesures sociales.

\section{Problèmes culturels prioritaires ${ }^{5}$}

Ainsi qu'en témoigne le tableau 5, nous retrouvons, dans les problèmes jugés prioritaires par les familles, une polarisation autour des enfants et des mères. Les intervenants, par contre, considèrent plutôt comme prioritaires des problèmes qui relèvent d'une dynamique de couple.

L'isolement et l'ennui sont au premier rang des problèmes culturels jugés prioritaires tant par les familles que par les intervenants. Le manque de soutien de la famille élargie, le fait que leurs enfants soient jeunes, les problèmes de garde, le climat, les problèmes financiers constituent un ensemble de facteurs qui contribuent à l'isolement des mères. L'isolement est toutefois fréquemment mentionné comme problème des femmes en général (Guberman et al., 1993). Néanmoins, les immigrantes se distinguent par le fait qu'elles méconnaissent souvent les services auxquels elles peuvent recourir, ou alors elles hésitent à le faire, car elles perçoivent ces problèmes comme relevant de la sphère privée, par ailleurs singulièrement rétrécie par la perte du réseau de soutien à la suite de la migration (Alvarado et Gravel, 1991; Duval, 1991).

5. Tout comme les problèmes sociaux, une liste des problèmes culturels était jointe au questionnaire. Cette liste comprenait: des problèmes liés à l'utilisation de la nourriture trouvée en société d'accueil; des problèmes vestimentaires liés au changement des saisons; des problèmes de garde des enfants; des problèmes liés à un contexte défavorable au développement des enfants; des problèmes liés à la socialisation des enfants ; des problèmes de discipline des enfants en société d'accueil ; des problèmes liés à la présence ou à l'absence de la parenté; des problèmes d'isolement; des problèmes liés à des rapports hommes-femmes différents; des problèmes liés à un nonpartage des tâches concernant la conduite du ménage et le soin et l'éducation des enfants; des problèmes liés au travail de la femme à l'extérieur du foyer ; des problèmes liés aux croyances religieuses des familles. 
Tableau 5

Hiérarchisation des problèmes culturels

par les familles et les intervenants

\begin{tabular}{lll}
\hline $\begin{array}{l}\text { Problèmes sociaux } \\
\text { jugés prioritaires }\end{array}$ & $\begin{array}{c}\text { Rang selon } \\
\text { familles }\end{array}$ & $\begin{array}{c}\text { Rang selon } \\
\text { intervenants }\end{array}$ \\
\hline
\end{tabular}

L'isolement et l'ennui

$1 \quad 1$

La garde des enfants

26

Le manque d'argent

3

Modalités d'éducation et de loisirs

des enfants

410

Le manque de travail et de formation au travail

Les rapports hommes-femmes

\section{1}

6

7

Le partage des tâches dans les soins, l'éducation des enfants et la conduite du ménage

Le manque de soutien de la parenté

La violence conjugale

$\begin{array}{ll}* & 3 \\ * & 4 \\ * & 5\end{array}$

* Ces problèmes n'ont pas été jugés prioritaires.

Les familles considèrent ensuite comme prioritaires les problèmes liés à la garde des enfants (deuxième rang, sixième rang pour les intervenants). Pour certaines, le problème de la garde des enfants relève $d^{\prime}$ une question financière et, pour $d^{\prime}$ autres, le recours à une gardienne non parente n'est pas acceptable. De nouveau, I'absence de la famille élargie implique une perte quant aux soins à donner aux enfants (Alvarado et Gravel, 1991; Legault et Lafrenière, 1992) et une augmentation des tâches dévolues aux mères (Meintel et al., 1985). Ainsi, les préoccupations liées aux enfants sont prioritaires pour les familles au cours des premières années, d'autant plus que ces enfants sont jeunes.

Si le manque de soutien de la parenté est très fréquemment rapporté par les familles, il est intéressant de noter que celles-ci ne jugent pas ce problème prioritaire. Nous pouvons penser qu'en termes de processus migratoire ces familles traversent ce qui est fréquemment qualifié de période de deuil. Plusieurs d'entre elles vivent en fait une période de transition entre la perte d'un réseau de parenté et la reconstitution d'un réseau d'entraide dans la société d'accueil.

Les familles placent aussi aux troisième et cinquième rangs certains besoins essentiels, tel le manque d'argent et de travail. Le fait 
que ces problèmes soient retenus comme problèmes prioritaires, alors qu'ils ne sont pas des problèmes de type culturel, traduit leur importance dans le quotidien des familles. En ce sens, il y a concordance avec plusieurs de nos données sociodémographiques ayant trait au revenu et à la situation de travail des familles.

Les modalités d'éducation et de loisirs des enfants sont au quatrième rang des problèmes jugés prioritaires par les familles. Certaines familles craignent l'influence de la société d'accueil dans l'éducation de leurs enfants, d'autres évoquent plutôt le manque de loisirs pour eux. Sachant que pour $54 \%$ des familles, la recherche d'un meilleur futur pour les enfants était la ou une des raisons ayant motivé le projet migratoire, il devient plus aisé de comprendre ces appréhensions; les familles découvrent en effet un contexte peut-être moins favorable à l'éducation de leurs enfants que ce qu'elles imaginaient. Même si ces préoccupations ne sont pas exclusives au Québec, la situation des jeunes et les problèmes de décrochage scolaire, de violence et de drogues peut inquiéter sinon alarmer certaines familles immigrantes.

Les intervenants, pour leur part, placent ces préoccupations au dixième rang. Outre cette disparité dans l'importance accordée à la problématique des modalités d'éducation, les intervenants s'interrogent alors davantage sur la confrontation des modèles de relations parents-enfants, sur les modalités de discipline, sur l'éducation des enfants en général. Legault et Lafrenière (1992) associent ces attitudes à la notion de choc culturel intervenants-clients pouvant mener à des situations d'incompréhension interculturelle.

Les intervenants attachent par ailleurs beaucoup d'importance aux rapports hommes-femmes, puisqu'ils les placent au deuxième rang des problèmes culturels prioritaires. Les familles ne mentionnent pas ce type de problème. Elles s'expriment plutôt en termes de difficultés de couple ou de difficultés familiales, et ces problèmes arrivent au sixième rang des problèmes culturels prioritaires. De la même façon, les intervenants considèrent comme un problème prioritaire (au troisième rang) le non-partage des tâches liées à l'éducation des enfants et à la conduite du ménage, alors que les familles ne le retiennent pas comme problème prioritaire. Ce type de problème se retrouve au dix-huitième rang des problèmes le plus fréquemment rapportés par les familles. La question de la violence conjugale est aussi une préoccupation chez les intervenants, alors qu'elle n'est pas citée par les familles.

Ces choix de la part des intervenants témoignent de leur sensibilisation à la dynamique des couples immigrants et au phénomène 
de la violence faite aux femmes, thèmes abondamment traités dans les écrits (Beaulieu et Concha, 1988; Legault et Rojas-Benavente,1988; Freire, 1989; Barudy, 1992). Cette sensibilisation peut être associée au mouvement féministe occidental des dernières décennies, les intervenants de notre échantillon étant en majorité des femmes; celles-ci peuvent avoir été impliquées dans ce mouvement pour elles-mêmes et pour leurs clientes et, de ce fait, être plus préoccupées de notions d'égalité dans les rapports hommes-femmes. Cet ordre de priorité présuppose toutefois une remise en question et une renégociation des rôles sociaux dans le couple de la part des jeunes familles immigrantes. Nos données ne nous permettent pas de soutenir ou d'infirmer une telle remise en question même si, comme le sous-tendent les écrits, les problèmes conjugaux sont fréquents chez les jeunes familles immigrantes (Duval, 1991; Juteau, 1991). La sphère privée se limitant souvent au conjoint et aux jeunes enfants, on comprendra que le climat familial puisse être source de tensions favorisant l'émergence de conflits conjugaux et parentaux.

Pour interpréter cette divergence de priorités dans les problèmes culturels, nous nous interrogeons sur les réalités dont font état les intervenants et les familles. Les problèmes mis en avant par les intervenants seraient-ils davantage des problèmes rencontrés par les familles à un moment plus lointain de leur séjour au pays d'accueil, ou ces problèmes seraient-ils considérés comme secondaires par les familles, compte tenu des embûches quotidiennes qu'elles doivent affronter, ou encore ces problèmes seraient-ils considérés comme faisant partie de la sphère privée? Nous pouvons expliquer finalement cette divergence de hiérarchisation par le fait que les intervenants s'expriment en fonction de la clientèle qu'ils côtoient, alors que les familles traduisent une vision représentative de la population sélectionnée.

Soulignons par ailleurs que $54 \%$ des familles qui se sont exprimées sur un problème culturel qu'elles jugeaient prioritaire $n^{\prime}$ ont consulté personne à ce sujet. Cela soulève toute la question de l'adaptation et de l'adéquation des services sociaux aux difficultés culturelles mises en avant par les familles. Les migrants peuvent hésiter à recourir aux services existants pour les raisons mentionnées, c'est-à-dire la méconnaissance des services et la nature même du problème, pour lequel une consultation formelle est inappropriée, mais aussi parce que, dans le pays d'origine, le réseau de services publics était inexistant, ne répondait pas à ce type de problème ou était synonyme d'impasse. 


\section{CHEMINEMENT DES FAMILLES POUR LEUR PROBLÈME PRIORITAIRE}

Le tableau 6 résume le cheminement des familles pour leur problème prioritaire des secteurs social et culturel. Le taux de non-consultation pour les problèmes sociaux est de $41 \%$, alors qu'il s'élève à $54 \%$ pour les problèmes culturels. Au moment des consultations, les familles vont $\mathrm{d}^{\prime}$ abord et davantage vers le réseau formel ${ }^{6}$ pour le secteur social, alors qu'elles recourent plutôt au réseau informel pour le secteur culturel. La catégorie intermédiaire que forment les familles qui, dans un premier temps, se sont tournées vers le réseau informel pour ensuite consulter le réseau formel demeure peu importante, soit $5 \%$ pour le secteur social et $3 \%$ pour le secteur culturel. C'est donc dire qu'il y a concordance, chez les familles, entre le réseau d'appartenance des premières personnes souhaitées ${ }^{7}$ et celui des personnes effectivement consultées.

Tableau 6

Cheminement des familles pour leur problème jugé prioritaire

\begin{tabular}{|c|c|c|}
\hline $\begin{array}{l}\text { Cheminement des familles ayant } \\
\text { jugé un problème prioritaire }\end{array}$ & $\begin{array}{l}\text { Secteur } \\
\text { SOCIAL }\end{array}$ & $\begin{array}{l}\text { Secteur } \\
\text { CULTURE }\end{array}$ \\
\hline Aucune consultation & $41 \% \quad(104)$ & $54 \%(106)$ \\
\hline $\begin{array}{l}\text { Consultation dans réseau formel } \\
\text { seulement }\end{array}$ & $36 \%(91)$ & $17 \%(34)$ \\
\hline $\begin{array}{l}\text { Consultation d'abord dans réseau } \\
\text { informel, puis dans réseau formel }\end{array}$ & $5 \%(12)$ & $3 \%(6)$ \\
\hline $\begin{array}{l}\text { Consultation dans réseau informel } \\
\text { seulement }\end{array}$ & $15 \%(37)$ & $25 \%(50)$ \\
\hline Données manquantes & $3 \%(7)$ & $1 \%(2)$ \\
\hline Total & $100 \%(251)$ & $100 \%(198)$ \\
\hline
\end{tabular}

Il faut aussi noter que lorsqu'il n'y a pas eu de consultation pour un problème jugé prioritaire par la répondante, ce problème est demeuré non résolu dans un nombre élevé de cas. Cette proportion est de $49 \%$ pour le secteur social et de $60 \%$ pour le secteur culturel. Étant donné

6. Dans notre enquête le réseau formel de services comprenait l'ensemble des services professionnels offerts avec la carte d'assurance-maladie du Québec, de même que les services publics. Il comprenait également des professionnels qui, sans être des services publics, relevaient du réseau formel de services, tels que les dentistes, les avocats, les psychologues, etc. Quant au réseau informel, il se composait essentiellement de parents, d'amis ou de non-professionnels.

7. Les familles avaient la possibilité de se prononcer sur deux personnes de leur choix, quant à une consultation pour leur problème prioritaire. 
que pour le secteur social les problèmes prioritaires sont liés au manque de travail, aux problèmes financiers et aux difficultés de communication et que pour le secteur culturel ils sont liés à l'isolement et à l'ennui ainsi qu'à la garde des enfants, ces proportions de non-résolution de problèmes peuvent se révéler inquiétantes.

\section{Préférences de CONSUltation}

Les difficultés de disponibilité et d'accessibilité aux services sont traitées de façon indistincte dans plusieurs études (Valdez et al., 1993 ; Legault et Lafrenière, 1992; Urrutia-Rojas et Aday, 1991 ; Hulka et Wheat, 1985). Dans notre étude, nous les avons traitées séparément en nous enquérant d'abord des préférences de consultation des familles pour leurs problèmes sociaux et culturels prioritaires. Les intervenants se sont également exprimés sur ces préférences quant aux problèmes qu'ils estimaient prioritaires pour les familles.

De manière générale et ainsi que l'indique le tableau 7 , les familles souhaitent majoritairement recourir au réseau formel pour leurs problèmes sociaux. Les intervenants sont du même avis, en proportion plus élevée. Pour le secteur culturel, alors que les familles souhaitent consulter surtout des personnes du réseau informel, les intervenants croient toujours que la consultation de personnes du réseau formel est souhaitable, quoique dans des proportions moins affirmées que pour le secteur social.

TABLEAU 7

Réseau d'appartenance des premières personnes dont la consultation est souhaitée

\begin{tabular}{|c|c|c|c|c|}
\hline \multirow[b]{2}{*}{ Réseau d'appartenance } & \multicolumn{2}{|c|}{ Secteur SOCIAL } & \multicolumn{2}{|c|}{ Secteur CULTUREL } \\
\hline & familles & intervenants & familles & intervenants \\
\hline Réseau formel & $64 \% \quad(110)$ & $75 \% \quad(27)$ & $35 \%(37)$ & $59 \%(20)$ \\
\hline Réseau informel & $36 \%(62)$ & $25 \%$ & $65 \%(68)$ & $41 \%(14)$ \\
\hline Total & $100 \%(172)$ & $100 \%$ & $100 \%(105)$ & $100 \%$ \\
\hline
\end{tabular}

Pour leur problème social prioritaire (le travail, la communication, le manque d'argent, le logement), $64 \%$ des familles qui se sont prononcées souhaitent consulter le réseau formel, c'est-à-dire un agent ou un conseiller en emploi ou un établissement d'enseignement. Les intervenants estiment aussi que les familles souhaitent majoritairement (75\%) recourir au réseau formel, en privilégiant toutefois les intervenants 
communautaires et les travailleuses sociales, c'est-à-dire des intervenants travaillant dans les établissements où ils travaillent eux-mêmes.

Le pourcentage de personnes ayant jugé prioritaire un problème social, mais n'ayant souhaité consulter personne, est de $32 \%$. Cette tendance s'accentue davantage lorsqu' on considère le secteur culturel. En effet, près de la moitié des familles (47\%) qui ont reconnu l'importance d'un problème culturel ne souhaitent consulter personne pour ce problème ${ }^{8}$. Pour ce qui est de leurs problèmes prioritaires du secteur culturel, c'est-à-dire l'isolement et l'ennui, la garde des enfants, le manque d'argent et de travail et les modalités d'éducation des enfants, les familles souhaitent consulter majoritairement le réseau informel. D'après les intervenants, les familles souhaiteraient consulter et consulteraient toujours davantage le réseau formel, c'est-à-dire une garderie, un agent ou un conseiller, une travailleuse sociale pour les problèmes culturels qu'eux jugent prioritaires, c'est-à-dire l'isolement et l'ennui, les rapports hommes-femmes inégalitaires, le non-partage des tâches, le manque de soutien de la parenté et la violence conjugale.

$\mathrm{Si}$, dans ce secteur, la disparité entre les intervenants et les familles est frappante, elle se comprend au regard des problèmes considérés comme prioritaires par les uns et par les autres. Ainsi les familles qui estiment prioritaires I'isolement et l'ennui, la garde des enfants, les modalités d'éducation et de loisirs sont des familles récemment immigrées qui souhaitent discuter de ces problèmes avec des membres de la famille, des voisins, des amis, car elles ont l'habitude de consulter leur réseau (informel) pour ce type de problèmes. De la même façon, les intervenants, en raison du type de problèmes culturels qu'ils jugent prioritaires, auront tendance à suggérer une consultation du réseau formel, car, pour eux, ce réseau est le plus susceptible d'aider les familles à régler ces problèmes.

\section{Disponibilité des personnes souhaitées}

Les préférences de consultation pour les réseaux formel et informel se confirment au moment des consultations. Dans le secteur social, les familles qui souhaitaient consulter majoritairement le réseau formel le font dans une proportion de $61 \%$. Les intervenants, pour leur part, estiment que les familles le font dans $83 \%$ des cas. Quant au secteur culturel, alors que les familles souhaitaient davantage consulter le réseau informel (dans $65 \%$ des cas), elles le font dans des proportions

8. Quarante-sept pour cent $(47 \%)$ des familles qui ont jugé prioritaire un problème culturel ne souhaitent consulter personne. Nous avons vu que cette proportion s'élève à $54 \%$ lorsqu'on considère les personnes souhaitées et non consultées de fait. 
semblables $(66 \%)$. Les intervenants estiment plutôt que les familles consultent le réseau formel (dans $59 \%$ des cas) pour ce secteur. Dans l'ensemble, le réseau informel est plus utilisé par les familles dans les secteurs social et culturel que ce qu'estiment les intervenants. Nous croyons cette divergence d'opinion entre les intervenants et les familles liée aux différents problèmes jugés prioritaires par les uns et par les autres.

Le taux de non-consultation des personnes souhaitées est relativement faible 9 . Ces proportions sont de $29 \%$ pour le secteur social et de $22 \%$ pour le secteur culturel d'après les familles et de $14 \%$ et $12 \%$ selon les intervenants.

\section{LIEUX DE CONSULTATION DES PERSONNES DU RÉSEAU FORMEL}

Lorsqu'on s'attarde maintenant aux consultations effectuées, il est étonnant de constater les écarts de perception entre les intervenants et les familles quant aux lieux de consultation. Ainsi que nous I'indique le tableau 8, pour les deux secteurs, les intervenants citent les CLSC comme premier lieu de consultation, alors que les familles indiquent des lieux distincts selon les problèmes.

Pour les problèmes sociaux, les familles consultent principalement le bureau d'emploi et d'immigration, puis l'école et les organismes de logements à prix modique. Au troisième rang, elles consultent le bureau d'aide sociale, suivi des organismes communautaires. Ces choix de lieux se comprennent au regard des problèmes prioritaires des familles, soit les problèmes liés au manque et aux conditions d'accès au travail, les problèmes de communication et les problèmes financiers. Selon les intervenants, le CLSC serait le lieu de consultation le plus fréquenté par les familles, suivi du bureau d'emploi et des organismes communautaires. Cette importance accordée au CLSC est surprenante lorsqu'on s'attarde aux problèmes prioritaires des familles selon les intervenants, soit les problèmes financiers, les problèmes liés au manque de travail et les problèmes liés au statut d'immigration. Même

9. Pour les répondantes qui ont souhaité consulter quelqu'un, le taux de non-consultation est effectivement faible, ce qui veut dire que les répondantes qui se prononcent sur leurs préférences de consultation obtiennent, dans une large part, satisfaction. Ces taux de non-consultation sont toutefois plus élevés lorsqu'on inclut les répondantes qui n'ont souhaité consulter personne. Ainsi, à titre d'exemple, pour le secteur social le taux de non-disponibilité des personnes souhaitées est de $29 \%$, alors que le taux de répondantes n'ayant souhaité consulter personne est de $32 \%$, ce qui fait un taux de non-consultation global de $41 \%$. 
si l'on se réfère aux hypothèses déjà évoquées, soit les problèmes différents jugés prioritaires par les uns et les autres ou encore au fait que les intervenants ont tendance à faire référence à leurs propres lieux de travail (65\% travaillent en CLSC), il demeure difficile d'expliquer leur choix du lieu de consultation pour les problèmes sociaux. À moins qu'ils ne voient le CLSC comme un service de première ligne qui dirige la clientèle vers les autres services appropriés, en rapport avec son rôle officiel.

En ce qui a trait aux problèmes culturels, les garderies et les écoles, le CLSC et les organismes communautaires sont les lieux les plus fréquemment cités par les familles, alors que les intervenants citent de nouveau les CLSC, puis les organismes communautaires et divers autres lieux (maison, rue, etc.). Les visions des uns et des autres se rejoignent dans ce secteur, malgré une hiérarchisation différente des problèmes.

\section{Tableau 8}

Lieux de consultation

\begin{tabular}{lcccc}
\hline $\begin{array}{l}\text { Lieux de consultation } \\
\text { (réponses multiples) }\end{array}$ & \multicolumn{2}{c}{ Secteur SOCIAL } & \multicolumn{2}{c}{ Secteur CULTUREL } \\
familles & intervenants & familles & intervenants \\
\hline $\begin{array}{l}\text { Organisme } \\
\text { communautaire }\end{array}$ & $12 \%(14)$ & $21 \%(9)$ & $16 \%(9)$ & $25 \%(11)$ \\
Organisme religieux & $3 \%(3)$ & - & $9 \%(5)$ & $7 \%(3)$ \\
Bureau privé & $5 \%(6)$ & - & - & $2 \%(1)$ \\
CLSC & $3 \%(3)$ & $30 \%(13)$ & $23 \%(13)$ & $34 \%(15)$ \\
Clinique d'hôpital & - & - & $2 \%(1)$ & - \\
CSS & - & $2 \%(1)$ & - & $7 \%(3)$ \\
$\begin{array}{l}\text { Bureau d'emploi } \\
\text { et immigration }\end{array}$ & $42 \%(50)$ & $23 \%(10)$ & $11 \%(6)$ & - \\
Bureau d'aide sociale & $13 \%(15)$ & $5 \%(2)$ & $7 \%(4)$ & - \\
Services juridiques & - & $7 \%(3)$ & $2 \%(1)$ & $2 \%(1)$ \\
Autres & $24 \%(28)$ & $12 \%(5)$ & $32 \%(18)$ & $23 \%(10)$ \\
Total & $\mathbf{1 0 0 \% ( 1 1 9 )}$ & $\mathbf{1 0 0} \%(\mathbf{4 3 )}$ & $\mathbf{1 0 0 \% ( 5 7 )}$ & $\mathbf{1 0 0 \% ( 4 4 )}$
\end{tabular}




\section{ACCESSIBILITÉ AUX SERVICES SOCIAUX ${ }^{10}$}

La disparité familles-intervenants est frappante en ce qui a trait au pourcentage de personnes du réseau formel ayant présenté des difficultés d'accessibilité. Ces dernières sont présentes dans $29 \%$ (secteur social) et $32 \%$ (secteur culturel) des cas pour les personnes consultées du réseau formel, alors que, selon les intervenants, elles sont présentes respectivement dans $72 \%$ et $59 \%$ des cas. II nous semble ainsi que les intervenants ont largement intégré les contenus et critiques des différentes études et analyses portant sur l'accessibilité des services aux communautés culturelles (Lebel, 1986; Kein et Garon, 1987; Bisaillon, 1988 ; MSSS, 1990). Peut-être sont-ils aussi plus sévères et exigeants que les familles quant à leurs services qu'ils ne jugent pas assez accessibles? (Glampson et Goldberg, 1976).

Pour le secteur social en particulier, nous ajouterons que les services auxquels les familles affirment accéder sans difficulté ne sont pas des services sociaux comme tels, puisqu'il s'agit des services liés à l'immigration, à l'emploi, à l'aide sociale ou aux établissements d'enseignement. On pourrait toutefois concevoir ces services comme des services sociaux au sens large et se réjouir du fait que les familles les perçoivent comme accessibles.

Lorsqu'on s'attarde aux difficultés d'accessibilité et qu'on les compare à l'aide du tableau 9 , on constate que ce sont les raisons administratives qui dominent pour le secteur social et les raisons linguistiques pour le secteur culturel. On peut penser que ces dernières sont celles qui correspondent au pourcentage des répondantes qui ne parlent ni le français ni l'anglais couramment, soit $19 \%$ et $24 \%$. Nous sommes assez près des pourcentages de $29 \%$ et $32 \%$ des répondantes ayant expérimenté des difficultés d'ordre linguistique dans I'accessibilité aux services. La prédominance de ce type de raisons pour le secteur culturel s'explique sans doute par la complexité des problèmes de ce secteur, complexité qui nécessite plus qu'une maîtrise de l'une des deux langues de la société d'accueil pour les exprimer.

Quant aux raisons administratives qui dominent dans le secteur social, elles correspondent aux démarches à entreprendre, aux horaires à connaître et à respecter, aux rendez-vous à prendre. Ce sont là des domaines dont plusieurs services sociaux sont conscients et qu'il

10. En ce qui regarde l'accessibilité aux services, notre enquête n'a porté que sur les difficultés d'accessibilité aux personnes du réseau formel. Pour ce qui est des personnes du réseau informel de services, seule leur disponibilité a été notée. 
importe de souligner sur le plan des améliorations souhaitables. L'étude de Waxler-Morrison et al. (1990) mentionne également les difficultés linguistiques, bureaucratiques et de pauvreté dans l'accès aux services. Il est aussi intéressant de constater que respectivement $7 \%$ (secteur social) et $21 \%$ (secteur culturel) des familles de notre échantillon signalent des difficultés économiques, alors que les services de santé et sociaux sont gratuits, à quelques exceptions près. Le pourcentage assez élevé de difficultés d'accessibilité liées au manque d'information (21\%, intervenants) et à la catégorie "autres raisons » $(29 \%$, familles) dans le secteur culturel renvoie de nouveau à la complexité de ce secteur. Il n'est pas évident en effet de savoir où s'adresser pour des problèmes d'ordre culturel et plusieurs types de difficultés peuvent jouer dans I'accessibilité au service approprié.

TABLEAU 9

Difficultés d'accessibilité des personnes consultées du réseau formel

\begin{tabular}{lcccc}
\hline $\begin{array}{l}\text { Lieux de consultation } \\
\text { (réponses multiples) }\end{array}$ & \multicolumn{2}{c}{ Secteur SOCIAL } & \multicolumn{2}{c}{ Secteur CULTUREL } \\
& familles & intervenants & familles & intervenants \\
\hline Économiques & $7 \%(6)$ & $19 \%(15)$ & $21 \%(6)$ & $14 \%(8)$ \\
Géographiques & $13 \%(11)$ & $1 \%(1)$ & - & $5 \%(3)$ \\
Administratives & $33 \%(29)$ & $30 \%(24)$ & $11 \%(3)$ & $11 \%(6)$ \\
Linguistiques & $17 \%(15)$ & $25 \%(20)$ & $32 \%(9)$ & $34 \%(19)$ \\
$\begin{array}{l}\text { Manque } \\
\text { d'information }\end{array}$ & $8 \%(7)$ & $15 \%(12)$ & $7 \%(2)$ & $21 \%(12)$ \\
Autres raisons & $22 \%(19)$ & $9 \%(7)$ & $29 \%(8)$ & $14 \%(8)$ \\
Total & $\mathbf{1 0 0} \%(\mathbf{8 7})$ & $\mathbf{1 0 0} \% \mathbf{( 7 9 )}$ & $\mathbf{1 0 0} \% \mathbf{( 2 8 )}$ & $\mathbf{1 0 0} \% \mathbf{( 5 6 )}$ \\
\hline
\end{tabular}

La question de l'accessibilité aux services sociaux et de santé est, à l'évidence, complexe et plusieurs domaines doivent être touchés pour l'améliorer. À titre d'exemple, nous citons diverses rubriques, identifiées par le CSSMM $^{11}$ en 1990, à examiner pour une meilleure accessibilité des services: les mandats, les objectifs et rôles des organismes, les structures et le fonctionnement, les politiques et programmes, I'organisation des services, la formation, la sensibilisation et le perfectionnement des intervenants, I'accueil et l'adaptation des services, l'embauche.

11. Document de référence pour la préparation du plan d'action du CSSMM à I'intention des communautés culturelles (1990). 
Tous ces domaines, croyons-nous, doivent être abordés dans une perspective de changement organisationnel qui rende les services sociaux accessibles.

\section{COMPATIBILITÉ DES SERVICES SOCIAUX}

Les perceptions des familles et des intervenants quant à la compatibilité des services de santé et des services sociaux sont divergentes en plusieurs points, la vision des intervenants étant plus critique ${ }^{12}$.

\section{Difficultés d'interprétation et de communication ${ }^{13}$}

Bien qu'au chapitre de l'accessibilité des services (secteur culturel), les familles aient placé au premier rang les difficultés linguistiques, ces mêmes services ne semblent pas pour autant incompatibles, puisque les familles manifestent très peu ( $9 \%$ dans le secteur social et $6 \%$ dans le secteur culturel) le besoin de recourir à un interprète lors des consultations ${ }^{14}$. Serait-ce que les familles estiment qu'elles peuvent ou doivent se débrouiller sans interprète? Les intervenants soutiennent d'autre part massivement ( $77 \%$ ) que les familles ont besoin d'un interprète lors des consultations. Dans leur étude, Waxler-Morrison et al. (1990) déplorent que plusieurs intervenants sous-estiment la capacité des familles à comprendre et à s'exprimer en anglais. Nous nous interrogeons sur ce phénomène à la fois contradictoire et généralisé. Les intervenants auraient-ils à ce point intégré les recommandations quant à l'accessibilité linguistique des services qu'ils surestiment la nécessité de services d'interprétariat ou peut-être se sentent-ils plus adéquats et compétents professionnellement s'ils peuvent s'assurer les services d'un interprète?

12. Mentionnons que les intervenants se sont prononcés sur la compatibilité des services pour les deux secteurs combinés, alors que les familles l'ont fait de manière distincte. Il en résulte un commentaire global de la part des intervenants et, de ce fait, moins nuancé.

13. Ces difficultés font référence au besoin d'un interprète, à la disponibilité de cet interprète et aux problèmes liés au processus d'interprétation.

14. Toutefois, lorsqu'un interprète était souhaité, celui-ci était souvent non disponible. Lorsqu'il était disponible, les répondantes disent avoir éprouvé des difficultés d'interprétation dans la majorité des cas. Notons toutefois qu'il s'agit ici d'un nombre très restreint de cas. Par exemple, dans le secteur culturel, $6 \%(3 / 50)$ des répondantes ont déclaré avoir eu besoin d'un interprète et, de ce nombre, dans $67 \%$ (2/3) des cas, un interprète n'était pas disponible. 
II reste qu'un écart important existe entre notre échantillon où le tiers des répondantes parlent peu ou pas le français ou l'anglais et leur opinion quant au besoin d'un interprète. Peut-être les services existants offrent-ils déjà des services d'interprétariat satisfaisants ou encore les professionnels maîtrisent-ils les langues d'usage des répondantes. Nous pourrions aussi faire l'hypothèse que la clientèle utilisatrice des services a plus de difficultés linguistiques que la population de l'échantillon.

\section{Difficultés liées aux relations humaines}

Les intervenants sont, encore une fois, plus critiques que les familles quant à la qualité des relations humaines lors des consultations. Nous faisons référence, en relation avec ces difficultés, au temps insuffisant accordé au moment des consultations, au fait de n'avoir pu consulter un intervenant ou une intervenante de la même culture ou parlant la même langue que soi ou encore au fait d'avoir été dérangé par des différences d'âge ou de sexe entre soi et l'intervenant ou l'intervenante lors des consultations. Les intervenants ont-ils de nouveau intégré une critique des services que les familles ne semblent pas partager? Certains écrits nous incitent à considérer le niveau d'éducation comme un élément déterminant de ces critiques. Dans une étude française (Leloup et al., 1989) portant sur les opinions des familles immigrantes à l'égard des services des institutions sanitaires, les auteurs ont pu noter que ces familles, issues de milieux défavorisés, avaient des opinions plus sévères. Les familles de notre échantillon, qui ont un niveau élevé d'éducation, soit se tirent mieux d'affaire, soit sont tolérantes et flexibles devant les services offerts.

Par ailleurs, les écrits sont éloquents sur les avantages et les inconvénients associés au fait d'appartenir à la même culture ou de parler la même langue que celle des clients (Welin et Ervin, 1991; Lebel, 1986). Certains clients préfèrent consulter un membre de leur communauté, estimant qu'ils pourront s'exprimer davantage et mieux se faire comprendre. Certains autres préféreront au contraire consulter quelqu'un qui n'appartient pas à leur communauté, craignant que leurs problèmes circulent dans la communauté ou qu'ils soient jugés.

Il est difficile de porter un jugement sur les difficultés de compatibilité, puisque tous ces facteurs peuvent jouer simultanément dans une consultation ou au contraire s'atténuer. Peu d'études ont traité simultanément de ces divers facteurs de compatibilité des services (âge, sexe, explications quant à la nature et au traitement des problèmes), si ce n'est l'étude de Flaskerud (1986) en association avec les services 
de santé mentale prodigués à certaines communautés culturelles aux États-Unis. La principale limite de cette étude réside dans le fait qu'il n'existe pas de pondération validée des différents facteurs énumérés, pondération qui permettrait de construire un indice de compatibilité culturelle des services.

En ce qui a trait aux différences d'âge et de sexe en tant que facteurs perturbateurs lors des consultations, on note que peu ou pas de répondantes affirment avoir été dérangées par ces facteurs; $d$ 'après les intervenants, au contraire l'âge de l'intervenant dérangerait les clientèles immigrantes dans $53 \%$ des cas et le sexe, dans $85 \%$ des cas. Cet écart de perception est possiblement lié au contexte dans lequel se situent répondantes et intervenants. Pour le secteur social, la nature des problèmes jugés prioritaires (travail, communication, problèmes financiers, etc.) et, conséquemment, le type de consultation effectuée ne donnent pas lieu de penser que l'âge ou le sexe de l'intervenant puisse interférer. Quant au secteur culturel, toutefois, on aurait pu s'attendre à y trouver certains problèmes de compatibilité des services lorsqu'on considère la nature des problèmes jugés prioritaires, soit ceux liés à l'isolement, à la socialisation et à l'éducation des enfants. Cependant, à la lumière des lieux de consultation fréquentés par les familles, soit les garderies, les établissements d'enseignement, les CLSC, les organismes communautaires, nous pouvons penser que les mères y ont rencontré un personnel essentiellement féminin avec qui elles ont pu se sentir en confiance ou encore qu'elles ont peu abordé, avec ces personnes rencontrées, des problèmes à caractère personnel.

\section{Difficultés liées au jugement professionnel ${ }^{15}$}

Les remarques et commentaires précédents valent également pour les questions portant sur le jugement professionnel. La vision des intervenants est, encore une fois, plus sévère que celle des familles et va dans le même sens que celle rapportée par l'étude menée auprès de plusieurs intervenants par la Table de concertation auprès des réfugiés (1984). Dans cette étude, les auteurs déplorent la méconnaissance des services, le mauvais acheminement des cas, la compétition entre les services et la sous-utilisation des organismes communautaires.

15. Ce type de difficultés renvoie essentiellement à une solution proposée qui correspond au problème présenté. 


\section{LIMITES DE L'ÉTUDE}

Comme nous le disions précédemment, s'il demeure possible d'étendre nos résultats à la population de jeunes familles immigrantes dans son ensemble, ces généralisations doivent être faites avec prudence. Outre les limites imposées par la taille de l'échantillon, le pouvoir de généralisation de notre étude est limité par le fait que notre échantillon de familles d'immigration récente devait, notamment, habiter les territoires des trois CLSC partenaires de l'étude. En ce sens, notre échantillon diffère, par certaines caractéristiques, de la population des jeunes familles immigrantes de la région de Montréal et, à plus forte raison, du Québec et d'ailleurs au Canada. Quant aux familles introuvables, bien que la plupart aient dû quitter la région de Montréal, la fraction qui y est demeurée ne nous est pas connue. Finalement, les caractéristiques des familles qui ont refusé de participer à l'étude nous sont inconnues.

La comparaison effectuée tout au long de l'étude entre les perceptions des familles et les perceptions des intervenants nous amène aussi à énoncer certaines réserves. Les familles, dans leurs réponses, se référaient à leur expérience personnelle vécue au cours de l'année précédant l'entrevue, alors que les intervenants se référaient à leur expérience clinique de pratique au cours de la même année. Ces derniers ont nécessairement une perspective de clientèle qui diffère de celle d'un échantillon de population. Il faut toutefois situer notre étude dans un contexte de connaissance des besoins de la population, connaissance sur laquelle reposent les mandats des CLSC et de la Direction de la santé publique. Cette recherche représente une contribution à cette tâche et ne minimise nullement l'importance d'études de clientèles qui devraient la compléter.

Les intervenants rencontrés travaillent dans certaines structures du réseau, tels les CLSC et les organismes communautaires. L'échantillon n'est donc pas représentatif de l'ensemble des intervenants du réseau québécois de la santé et des services sociaux et les perceptions recueillies leur sont propres.

Une autre limite de l'étude a trait à la complexité des problématiques abordées dans le secteur culturel. L'instrument privilégié pour la collecte des données, soit le questionnaire à questions fermées, de même que la brièveté de l'entrevue par une intervieweuse inconnue de la famille, a fait que certains problèmes ont été peu ou pas mentionnés, tels les problèmes de couple ou de violence conjugale, par ailleurs mentionnés par les intervenants. La comparaison entre la perspective des familles et la perspective des intervenants a pu en être affectée $d^{\prime}$ autant. 
Notons finalement que l'importance des problèmes économiques et de travail a pu reléguer au deuxième plan ou voiler des difficultés qui seraient d'un autre ordre. En dernier lieu, I'approche quantitative n'a pas permis l'approfondissement de certaines problématiques émergeant de l'étude, approfondissement que faciliterait une approche qualitative.

\section{CONCLUSION}

En conclusion, deux types de remarques se dégagent de façon assez claire et évidente de l'étude, soit: 1 . des remarques ayant trait à la composition sociodémographique des jeunes familles; 2 . des remarques spécifiques liées aux secteurs social et culturel.

D'un point de vue sociodémographique, les jeunes familles immigrantes rencontrées correspondent, si l'on se fie aux énoncés de nos politiques d'immigration, quasi en tous points au profil d'immigrants recherchés. Ce sont de jeunes familles, ayant de jeunes enfants, avec un niveau d'éducation enviable et des habiletés à intégrer le marché du travail à partir d'occupations compatibles exercées dans le pays d'origine. Ce sont aussi des familles qui possèdent une bonne connaissance du français. Or, on observe que ces familles «idéales » éprouvent de nombreuses difficultés d'adaptation et d'intégration au cours de leurs premières années au Québec: ces familles sont pauvres et la moitié d'entre elles ne réussissent pas à intégrer le marché du travail. Ces deux constatations, forcément très corrélées, ne peuvent entraîner que déception, voire humiliation. La vulnérabilité ainsi créée ne peut que mettre en péril le nouveau projet de vie de ces familles.

Faut-il en conclure qu'il y a hiatus entre nos politiques d'immigration et la concrétisation de ces politiques auprès de familles précises? Réussirons-nous à garder et à intégrer, avec tout leur potentiel, ces familles qui correspondent si bien au profil recherché? Les services de santé et les services sociaux ne réussissent par ailleurs que partiellement à répondre à leurs besoins immédiats. Les mandats de ces services ne devraient-ils pas être élargis pour apporter des réponses plus satisfaisantes aux familles récemment immigrées?

Nous dirions d'emblée que les jeunes familles immigrantes rencontrent des problèmes que l'on trouve dans l'ensemble de la population et, à ce titre, ces problèmes n'ont rien de particulier. Ils sont toutefois caractéristiques par leur ampleur et par la menace qu'ils constituent à l'intégration de ces familles. 
En ce qui a trait à la définition des problèmes, nous avons été frappés par le fait que les familles, dans le secteur culturel, insistent surtout sur les problèmes de manque de socialisation de leurs jeunes enfants et sur l'absence de contexte favorable à leur développement. Or, les intervenants mentionnent des problèmes liés à la dynamique du couple et à l'isolement des mères comme problèmes prioritaires. Cette différence de perception des problèmes entre les intervenants et les familles est d'ailleurs l'un des traits saillants de notre étude.

Que faut-il en conclure? Que des programmes susceptibles de répondre aux problèmes mentionnés doivent viser prioritairement les enfants tout en ayant une dimension qui permette $d^{\prime}$ impliquer les mères et leurs problèmes particuliers? Ces programmes, centrés sur les enfants et leur développement, donneraient aux mères une occasion de socialisation et d'ouverture les aidant à mieux se familiariser avec les mécanismes, les ressources et l'univers culturel de la société d'accueil.

Pour ce qui est des services, nous avons constaté que l'urgence des problèmes liés au travail ou à l'accès au travail domine dans le secteur social. L'emploi étant un secteur différent et hors du mandat des services sociaux, il nous est difficile de nous prononcer sur les besoins en services sociaux proprement dits. Ces besoins sont en quelque sorte masqués par les problèmes d'accès à l'emploi omniprésents dans notre étude.

Dans le secteur culturel, la notion de consultation est beaucoup moins évidente et on peut penser que les nouveaux arrivants doivent avoir atteint une phase ultérieure d'adaptation à la société d'accueil pour considérer la consultation dans ce secteur. Rappelons qu'un fort pourcentage de familles iront plutôt vers le secteur informel pour y recevoir de l'aide ou encore ne consulteront personne. Pour un important pourcentage de familles ayant jugé prioritaires ce type de problèmes, ceux-ci demeureront non résolus dans plus de $60 \%$ des cas.

Terminons par une idée créatrice d'intégration issue de nos contacts avec les familles, soit un jumelage précoce familles immigrantes - familles de la société d'accueil qui permettrait un accompagnement régulier et soutenu des nouvelles familles au cours de leurs premiers mois. Ces jumelages existent déjà sur une petite échelle; il y aurait lieu de les amplifier, d'en réactiver d'autres et surtout d'en trouver les modalités les plus prometteuses. Il y aurait lieu aussi, à travers ces jumelages, de favoriser la création de liens positifs entre nouveaux arrivants et citoyens de la société d'accueil en fonction de critères précis. Ces jumelages auraient également l'avantage de favoriser un processus dans les deux sens, c'est-à-dire une adaptation réciproque de la société d'accueil et des nouveaux arrivants. 


\section{Références bibliographiques}

Alvarado E. et S. Gravel (1991). Problèmes de stimulation et de développement des jeunes enfants issus de familles latino-américaines du quartier Côte-des-Neiges. DSC Sainte-Justine.

BAillargeOn, M. (1989). Population immigrée dans les régions métropolitaines du Québec et dans certaines municipalités du Grand Montréal, par pays de naissance. Recensement 1986: document 5, ministère des Communautés culturelles et de I'Immigration, Direction des études et de la recherche, Québec.

BARUDY, J. (1992). "Migration politique, migration économique: une lecture systémique du processus d'intégration des familles migrantes », Santé mentale au Québec, vol. XVII, n 2: 47-70.

BeAulieu, A. et M.E. CONCHA (1988). Les Latino-Américains au Québec, portrait des familles de Côte-des-Neiges. CLSC Côte-des-Neiges et Association des immigrants latino-américains de Côte-des-Neiges.

BEISER, M. et al. (1988). Puis... la porte s'est ouverte. Problèmes de santé mentale des immigrants et des réfugiés. Rapport du groupe chargé d'étudier les problèmes de santé mentale des immigrants et des réfugiés au Canada.

BIBEAU, G. et al. (1992). La santé mentale et ses visages. Un Québec pluriethnique au quotidien. Boucherville: Gaëtan Morin.

BISAILLON, H. (1988). Accessibilité des services sociaux du CSSMM aux communautés culturelles de son territoire. Document de travail, CSSMM.

CHOINIÈRE, R. (1990). La situation démographique et socio-économique de la population du Montréal métropolitain : principaux résultats du recensement de 1986 par CLSC et DSC. Regroupement de départements de santé communautaire du Montréal métropolitain.

DESJARDINS, D. (1990). Les communautés culturelles sur le territoire des CLSC Bordeaux-Cartierville et Saint-Laurent. Centre de recherche sur les services communautaires. Rapport de recherche.

DUVAL, M. (1991). Être mère au foyer et récemment immigrée à Montréal. Institut québécois de la recherche sur la culture.

FLASKERUD, J.H. (1986). "The Effects of Culture-Compatibility Intervention on the Utilization of Mental Health Services by Minority Clients», Community Mental Health Journal, vol. 22, nº $2:$ 127-141.

FREIRE, M. (1989). The Experiences of Refugee Families. Multiculturalism and Health Care: Realities and Needs. Proceedings of the Canadian Council and Multicultural Health. $1^{\text {st }}$ National Conference. Toronto, mars-avril, p. 95-96.

Glampson, A. et M. Goldberg (1976). "Post Seebohm Social Services: The Consumer's Viewpoint», Social Work Today, n $8: 9-14$.

GRAVEL, S. et D. GuAy (1990). Quelle population le CLSC Côte-des-Neiges rejoint-il ? DSC Sainte-Justine, CLSC Côte-des-Neiges.

GRES (1992). "Immigration et relations ethniques au Québec: un pluralisme en devenir », dans G. Daigle et G. Rocher, Le Québec en jeu. Montréal : PUM, p. 451-481. 
GuBERMAN, N. et al. (1993). Un mal invisible. L'isolement des femmes. Montréal: Remue-ménage.

Heneman, B., G. Legault, S. Gravel, S. Fortin et E. Alvarado (1994). Adéquation des services aux jeunes familles immigrantes. École de service social, Université de Montréal et Direction de la santé publique de Montréal, Rapport de recherche.

HULKA, B.S. et J.R. WHEAT (1985). "Patterns of Utilization; The Patient Perspective ", Medical Care, vol. 23, $\mathrm{n}^{\circ} 5: 438-459$.

Juteau, F. (1991). "Divorce and the Migration Process among Salvadorians in Montreal », dans S.P. Sharma, M. Ervin et D. Meintel (dir.), Immigrants and Refugees in Canada. Univ. of Sask., p. 282-293.

KEIN, D.A. et M. GARON (1987). Programmes d'accès à l'égalité et accès des minorités aux services publics. Commission des droits de la personne du Québec, Rapport de recherche.

LABELLE, M. (1989). "Immigration, main-d'œuvre immigrée et société d'accueil : réalités et contradictions », Nouvelles pratiques sociales, vol. 2, $\mathrm{n}^{\circ} 2$ : 106-122.

LEBEL, B. (1986). «Les relations entre les membres des communautés culturelles et les services sociaux et de santé », Canadian Ethnic Studies, vol. XVII, $\mathrm{n}^{\circ} 2: 79-89$.

LEGAULT, G. et E. RojAS-BenAVENTE (1988). «Femmes immigrantes et travail social: une recension des écrits », Service social, vol. 37, $\mathrm{n}^{\text {os }} 1-2: 176-196$.

LEGAULT, G. et M. LAFRENIÈRE (1992). Femmes, migrations, interventions : une rencontre interculturelle. École de service social, Université de Montréal, Rapport de recherche.

LEGAULT, G. et S. FORTIN (1994). «Adaptation-intégration des jeunes familles immigrantes: Cadre théorique et devis de recherche», Intervention, $\mathrm{n}^{\circ}$ 99: 49-62.

MEINTEL, D. et al. (1985). "La nouvelle double journée de travail des femmes immigrantes au Québec », Revue internationale d'action communautaire, vol. 14, n $54: 33-44$.

MCCI (Ministère des Communautés culturelles et de I'Immigration) (1990). L'intégration des immigrants et des Québécois des communautés culturelles: document de réflexion et d'orientation.

MSSS (Ministère de la Santé et des Services sociaux) (1990). Accessibilité des services aux communautés culturelles. Orientations et plan d'action. 1989-1991. Bureau des services aux communautés culturelles.

RENAUD, J. et al. (1993). Trois années d'établissement d'immigrants admis au Québec en 1989: Portrait d'un processus. Collection Études ethniques, $\mathrm{n}^{\circ} 5, \mathrm{MCCl}$.

TABLE DE CONCERTATION AUPRÈS DES RÉFUGIÉS (1984). La santé mentale des réfugiés.

URRUTIA-ROJAS, X. et L.A. ADAY (1991). "A Framework for Community Assessment: Designing and Conducting a Survey in a Hispanic Immigrant and Refugee Community », Public Health Nursing, vol. 8, $\mathrm{n}^{\circ} 1$ : 20-26.

VALDEZ, R.B. et al. (1993). "Insuring Latinos Against the Cost of Illness », JAMA, vol. 269, no $17: 889-894$. 
WAXLER-MORRISON, N., J. ANDERSON et E. RICHARDSON, dir. (1990). CrossCultural Caring, A Handbook for Health Professionals in Western Canada.

WELIN, L. et A.M. ERVIN (1991). "Refugee Clients and Social Service Agencies: Some Aspects of Cross-cultural Misunderstading », dans S. Sharma, A. Alexander, A.M. Ervin et D. Meintel (dir.). Immigrants and Refugees in Canada. Univ. of Sask., p. 178-184.

WILKINS, R. (1985). Données sur la pauvreté de la région métropolitaine de Montréal. DSC Hôpital général de Montréal. 